Check for updates

Cite this: Soft Matter, 2020,

16,1714

Received 29th October 2019,

Accepted 29th January 2020

DOI: $10.1039 / \mathrm{c} 9 \mathrm{sm} 02151 \mathrm{e}$

rsc.li/soft-matter-journal

\section{Translucent in air and iridescent in water: structural analysis of a salamander egg sac $\uparrow$}

\author{
Aleksandra V. Zabuga, ${ }^{a}$ Marcelle I. Arrigo, ${ }^{a}$ Jérémie Teyssier, (D) ${ }^{b}$ \\ Sébastien R. Mouchet, (D) ${ }^{c d}$ Kanto Nishikawa, (D) ${ }^{\text {e Masafumi Matsui, }}{ }^{\text {e }}$ \\ Miguel Vences (iD ${ }^{f}$ and Michel C. Milinkovitch (D) *a
}

Females of some Asian salamanders of the genus Hynobius deposit in streams their eggs embedded in a translucent envelope called an 'egg sac'. The edges of the envelope exhibit a spectacular blue-to-yellow iridescent glow, which instantaneously disappears when the sac is removed from water. First, our scanning electron microscopy analyses reveal that the inner surface of the $100 \mu \mathrm{m}$-thick envelope displays striations (length scale of about $3 \mu \mathrm{m}$ ), which are themselves covered by much smaller (190 $\pm 30 \mathrm{~nm}$ ) and quasi-periodic corrugations. The latter could constitute a surface diffraction grating generating iridescence by light interference. Second, our transmission electron microscopy and focused-ion-beam scanning electron microscopy analyses show that the bulk of the egg sac wall is composed of meandering fibres with a quasi-periodic modulation of $190 \pm 60 \mathrm{~nm}$ along the thickness of the envelope, generating a photonic crystal. Third, Fourier power analyses of 450 electron microscopy images with varying incident angles indicate that changing the surrounding medium from water to air shifts most of the backscattered power spectrum to the ultraviolet range, hence, explaining that the egg sac loses visible iridescence when removed out of the water. Fourth, the results of our photography and optical spectroscopy experiments of submerged and emerged egg sacs rule out the possibility that the iridescence is due to a thin film or a multilayer, whereas the observed non-specular response is compatible with the backscattering expected from surface diffraction gratings and volumetric photonic crystals with spatial 1D modulation. Finally, although we mention several potential biological functions of the egg sac structural colours and iridescence, we emphasise that these optical properties might be the by-products of the envelope material internal structure selected during evolution for its mechanical properties.

\section{Introduction}

The phenomenon of iridescence, i.e., the variation of colour of a material surface when observed and/or illuminated from different angles, is generally caused by interference of light reflected from periodic nanostructures. ${ }^{1-4}$ Because of recent advances in nanomaterial technologies, scientists are attempting to engineer artificial iridescent materials ${ }^{5-9}$ whose geometries are inspired from natural nanostructures evolved by living organisms during hundreds of millions of years of biological history: from photonic structures with 1D modulation (thin film, ${ }^{10}$ multilayers, ${ }^{11-13}$ and diffraction gratings ${ }^{14-16}$ ) in pigeon feathers, beetle elytra, colour changing copepods and iridescent snakes, to helicoid multilayers in the fruits of Pollia spp. ${ }^{17}$ and of Margaritaria nobilis, ${ }^{18}$ to chitinous gyroids on butterfly wings ${ }^{19}$ and guanine nanocrystal $3 \mathrm{D}$ lattices in the skin of chameleons. ${ }^{20}$

Here, we study an iridescence phenomenon occurring in the egg sacs of Asian salamanders of the family Hynobiidae; the eggs of these amphibians are laid embedded in elongated sacs of a jelly-like material and then externally fertilized. In various species $^{21}$ (e.g., the stream-breeding Hynobius amakusaensis, $H$. boulengeri, $H$. fuca, $H$. kimurae, $H$. naevius, $H$. shinichisatoi, H. ikioi, and H. tsuensis, and the pond-breeding Salamandrella keyserlingii), egg sacs are iridescent in water (Fig. 1a) but they immediately and completely loose this property when they are removed out of water (Fig. 1b). Fluid-induced colour changes have been explained by a reduction in the refractive index mismatch caused by the invasion of water in a porous photonic structure (as, e.g., in the elytra of the Dynastes hercules ${ }^{22}$ and Hoplia coerulea beetles ${ }^{22,23}$ ) or by structural modifications.

\footnotetext{
${ }^{a}$ Laboratory of Artificial and Natural Evolution (LANE), Department of Genetics and Evolution, University of Geneva, Sciences III, 30, Quai Ernest-Ansermet, 1211 Geneva 4, Switzerland. E-mail: Michel.Milinkovitch@unige.ch; Tel: +41223796785

${ }^{b}$ Department of Quantum Matter Physics, University of Geneva, Switzerland

${ }^{c}$ School of Physics, University of Exeter, Exeter EX4 4QL, UK

${ }^{d}$ Department of Physics, University of Namur, 5000 Namur, Belgium

${ }^{e}$ Graduate School of Human and Environmental Studies, Kyoto University, Kyoto, Japan

${ }^{f}$ Zoological Institute, Braunschweig University of Technology, Braunschweig, Germany

$\dagger$ Electronic supplementary information (ESI) available. See DOI: 10.1039/c9sm02151e
} 


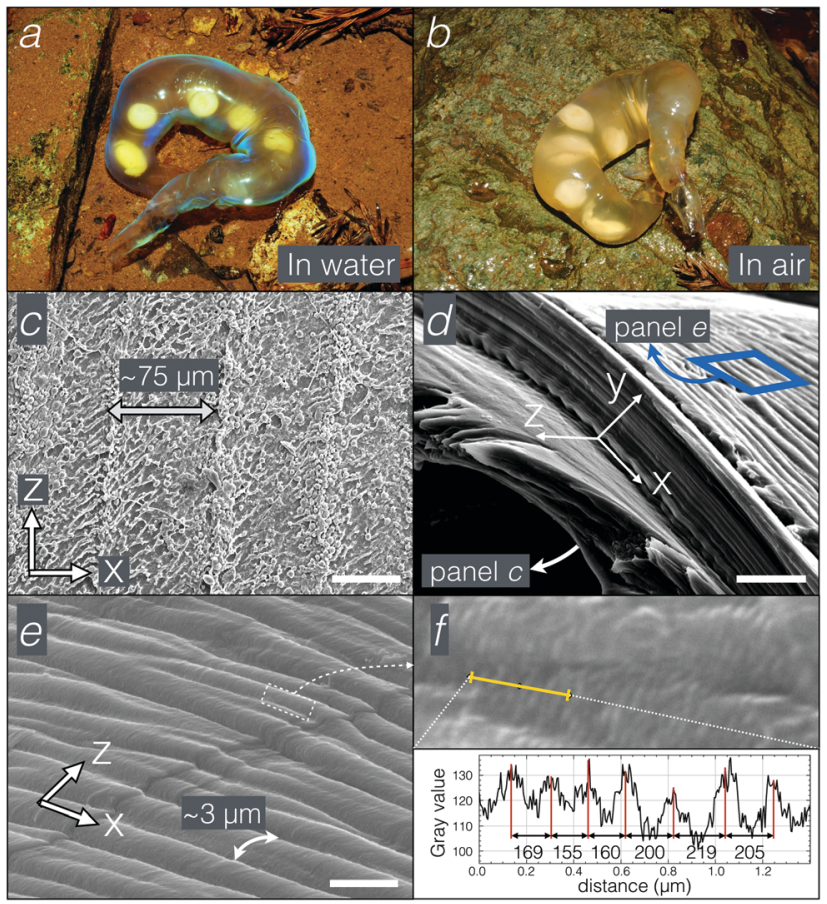

Fig. 1 ( $a$ and b) Photograph of a Hynobius kimurae egg sac in water (a) and outside of water (b) in its natural environment. (c-e) SEM micrographs of the outer surface (c), the cross-section (d), and the inner surface (e) of the egg sac. (f) SEM micrograph of the inner surface (upper panel) and profile of the corrugations (lower panel) along the line indicated in yellow. Scale bars: $50 \mu \mathrm{m}$ (c), $10 \mu \mathrm{m}$ (d), and $5 \mu \mathrm{m}$ (e).

The latter include the detachment of the photonic structure from initially connected absorbing layers as in drying Margaritaria nobilis fruit $^{18}$ and the decrease in interlamellar distance as in drying red alga Chondrus crispus. ${ }^{24}$ However, given that Hynobius egg sacs instantaneously lose their structural colours and iridescence when removed from water, the phenomenon cannot be due to drying/ wetting of the bulk of the egg sac material.

By combining optical measurements, microscopy and mathematical modelling, we show here that (i) the Hynobius kimurae (Hida salamander) egg sac wall is a 3D structure with a periodic 1D modulation causing iridescence, and (ii) removal of the egg sac from water causes a shift of the backscattered light to the UV range due to the exchange of surrounding media (from water to air) with very different refractive indices, hence, resulting in the loss of iridescence in the visible range. The function (if any) of the iridescence in Hynobius egg sacs is unknown. Further analyses are required to identify whether the structural colour and/or iridescence might attract male salamanders for external fertilisation, or constitute a visual signal to avoid cannibalism, or are mere by-products of egg sac material internal structures selected during evolution for their mechanical properties.

\section{Results and discussion}

Salamanders of the genus Hynobius breed in streams where the female lays one pair of envelopes, called 'egg sacs', that contain 8 to 212 eggs. ${ }^{25}$ The sac envelope is overall translucent, about
$0.1 \mathrm{~mm}$ thick, and its inner surface is coated with mucus. When the egg sac is submerged, its edges exhibit a spectacular blue glow (Fig. 1a), which instantaneously disappears when the sac is removed from water (Fig. 1b). We perform SEM (scanning electron microscopy) and TEM (transmission electron microscopy) analyses to characterise the structural properties of the sac envelope, as well as photography and optical spectroscopy of submerged and emerged egg sacs to record their optical response. Finally, we perform Fourier analysis of the actual 3D structure extracted from FIB-SEM (focused-ion-beam scanning electron microscopy) experiments.

\section{Egg sac ultrastructure}

The chemical composition of the egg sac is unknown. To characterise its structure, we used SEM, TEM, and FIB-SEM. All electron microscopy measurements were performed on samples fixed either in formalin (SEM) or in $2.5 \%$ glutaraldehyde (TEM and FIB-SEM). Note that formalin and glutaraldehydefixed samples are still iridescent, but not as strongly as the envelope of fresh sacs, possibly indicating a transformation of the material geometry due to fixation.

SEM micrographs of the egg sac outer surface reveal the presence of fibres and nodules (about 2-5 $\mu$ m-thick) covering $\sim 75 \mu \mathrm{m}$-wide periodic striations (Fig. 1c). This period is too large (compared to visible light wavelengths) to be involved in the observed photonic effect. The thickness of the egg sac wall is populated by a material that resembles a multilayer under SEM (Fig. 1d) while the inner surface is covered by a periodic pattern of striations with a length scale of about $3 \mu \mathrm{m}$ (Fig. 1e) and perpendicular to the outer surface with much larger striations. Higher magnification electron micrographs of the inner striations (Fig. 1f) reveal very thin and quasi-periodic corrugations (mean and standard deviation of $190 \pm 30 \mathrm{~nm}$ ) running perpendicular to the larger pattern.

In principle, the $190 \mathrm{~nm}$ corrugations on the inner side of the egg sac could constitute a surface diffraction grating and generate iridescence by light interference in the blue range of the visible spectrum. This scenario would however require the light to reach the inner side of the egg-sac wall without being affected by other nanostructures in the bulk of the material. Therefore, we conducted TEM analyses of the egg sac wall. Approximating the egg sac geometry as a cylinder (Fig. 2a), we define the $Y$-axis as the radial direction perpendicular to the wall, whereas the $X$ and $Z$ axes run along the circumference and the length of the cylinder, respectively. TEM images of $X Y$ and $Y Z$ sections (Fig. 2b-g) indicate that the egg sac wall is approximately $100 \mu \mathrm{m}$ thick and is covered by a 5 to $10 \mu \mathrm{m}$ electrondense outer layer (Fig. 2c). The latter progressively merges with the rest of the wall composed of quasi-periodic meandering fibres somewhat oriented perpendicular to the $X Y$ plane (Fig. 2d), i.e., parallel to the $Y Z$ plane (Fig. 2e and f), and with a length scale of about $140 \pm 40 \mathrm{~nm}$ (mean \pm standard deviation) determined using FFT (2D Fast Fourier Transform, see Methods) of TEM images in the $X Y$ plane (Fig. 2d).

To overcome the limitations of using a few TEM sections for inferring the geometry of the egg sac material, we used 


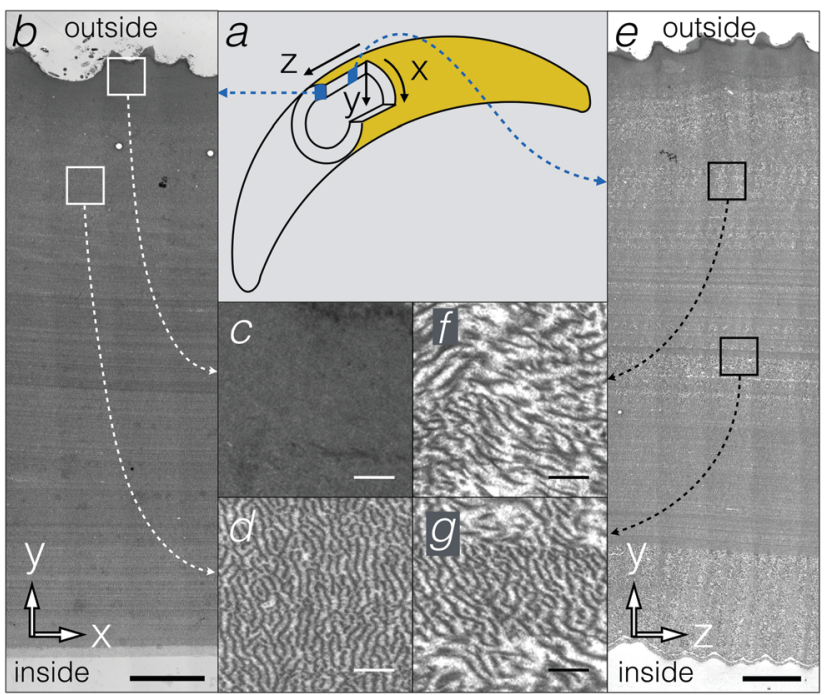

Fig. 2 (a) Schematic representation of the 3D coordinates of the egg sac. $(b-e)$ TEM micrographs of egg sac cross sections in the $X Y(b-d)$ and $Y Z$ (e-g) planes. Scale bars: $10 \mu \mathrm{m}$ (b and e), and $0.5 \mu \mathrm{m}$ (c, d, f and g).

FIB-SEM, a method that generates 3D geometry with nanometre resolution (Fig. 3a and Movie S1, ESI $\dagger$ ). The FIB-SEM data confirm that the undulating fibers in the bulk of the egg sac are about $50 \mathrm{~nm}$ thick and are preferentially oriented along the $Y Z$ plane (i.e., perpendicular to the $X Y$ and $X Z$ planes). This is quantitatively confirmed by FFT of FIB-SEM images of the $X Y$ and $X Z$ planes (Fig. $3 \mathrm{~b}$ and c) that reveal a quasi-periodic pattern with a length scale of $190 \pm 60 \mathrm{~nm}$ (mean \pm standard deviation). The difference of about $50 \mathrm{~nm}$ between the mean length scales derived from FIB-SEM versus TEM analyses might be due to the fact that the samples were cut from the inner versus outer curvature of the egg sac, respectively. As images of $Y Z$ planes do not exhibit order detectable with FFT (Fig. 3d), except in some localised areas (Fig. 2g), we suggest that the undulating fibres/surfaces form a 1D photonic crystal or, more specifically, a 3D material with a 1D modulation.

In addition to the modulation of electron density in the $X$-axis, the material forms layers (across the $Y$-axis, i.e., across the depth of the egg wall) of various thicknesses ranging from $350 \mathrm{~nm}$ to $1000 \mathrm{~nm}$. These layers correspond to slight but relatively sudden variation in the orientation of the modulation, hence, they are visible as somewhat undefined horizontal stripes of various shades of grey on TEM images of $X Y$ and $Y Z$ sections (Fig. 2b and e), and as a pseudo multilayer on the SEM images of cross-sections of the egg sac wall (Fig. 1d). Because these layers are not periodically distributed and are made of the same combination of electron-dense (fibres) and electron-transparent materials, they are unlikely to cause any photonic effect.

\section{Photography experiments}

We photographed the egg sac perpendicular to its $Z$-axis while illuminating the sac with a white light source positioned at $8^{\circ}$ away from the orientation of the camera (Fig. 4). By moving the egg sac horizontally (hence, along the $X$-axis) and perpendicular to the $Z$-axis, we gradually change the angle between the incident light and the normal to the egg sac surface from about $82^{\circ}$ (Fig. 4a) to $8^{\circ}$ (Fig. 4 b) to $-90^{\circ}$ (Fig. 4c). These experiments indicate that structural colours appear on the submerged egg sac surface when it is illuminated nearly tangentially (Fig. 4; middle panels). Note that the iridescent color is mostly blue in the outer curvature of the egg sac (Fig. 4a - middle panel) and spans a wider range of wavelengths (blue to green to yellow) in the inner curvature of the sac (Fig. 4c - middle panel). Structural colours disappear almost entirely when the sac is removed out of the water (Fig. 4 - lower panels). To confirm the effect of varying the angle of incident light, we performed additional experiments where we tangentially illuminated the

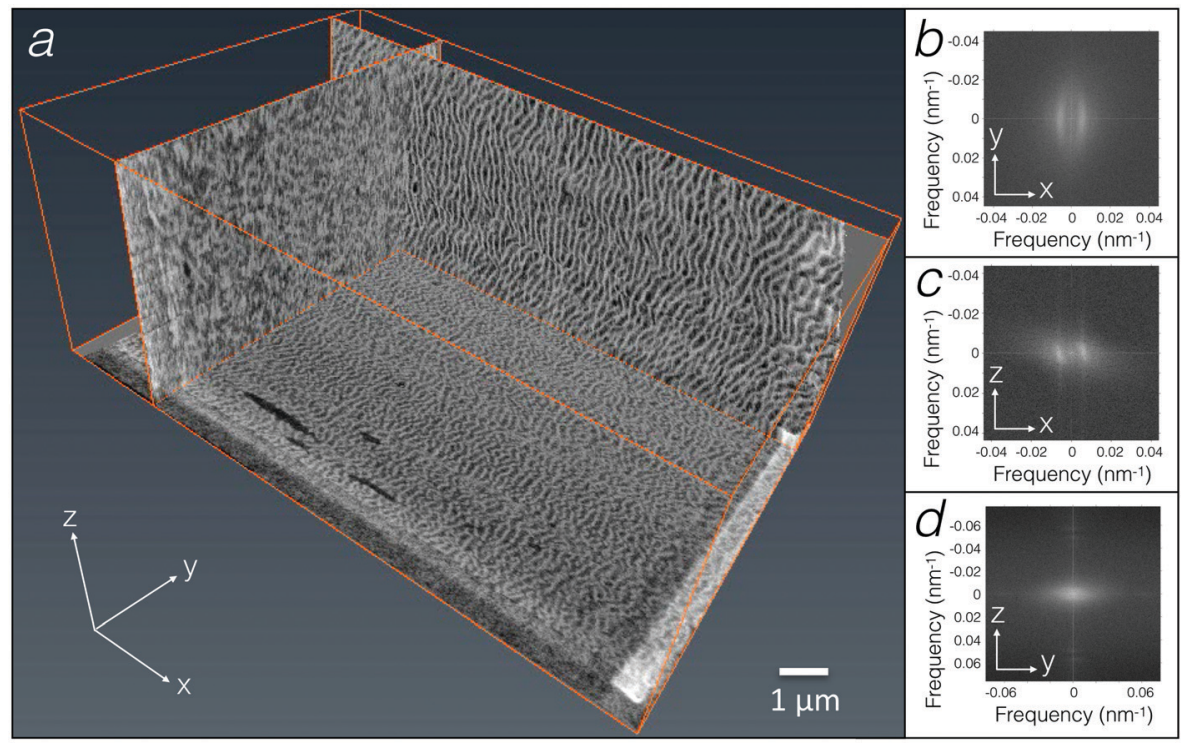

Fig. 3 (a) FIB SEM reconstructions of $X Y, Y Z$ and $X Z$ planes of the egg sac material. The stacking direction is along the $Z$-axis. (b-d) $2 D$ Fast Fourier Transform spectra of the egg sac in the $X Y, X Z$ and $Y Z$ planes. 


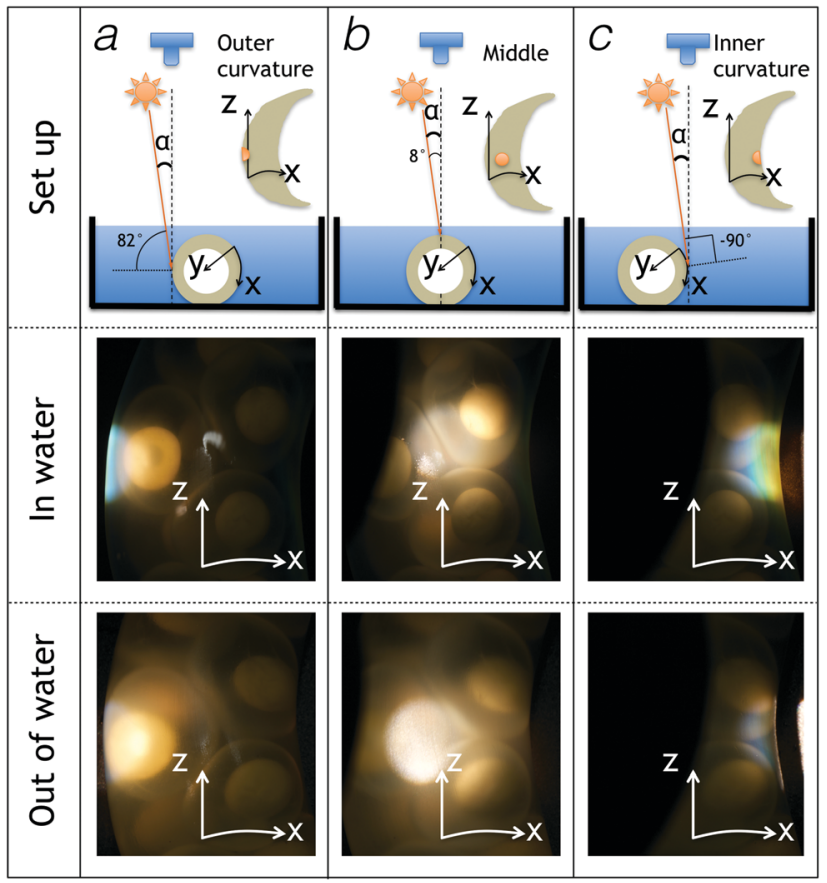

Fig. 4 Comparison of reflected colours on different parts of the egg sac in water and in air with a nearly normal light incidence $\left(\alpha=8^{\circ}\right)$. The position of the egg sac is changed relative to the light source and camera such that the outer curvature (a), the middle (b), and the inner curvature (c) of the egg sac are illuminated.

egg sac inner curvature with white light and changed the position of the camera as illustrated in Fig. S1a (ESI $\dagger$ ). The iridescent reflectance gradually fades out as the angle between the incident light and the camera $(\alpha+\beta$ in Fig. S1a, ESI $\dagger)$ increases from $8^{\circ}$ to $81^{\circ}$. These results confirm that reflectance is maximum at small angles between the incident light and the camera's objective and, hence, rule out the possibility that the Hynobius egg sac iridescence is caused by a thin film or a multilayer. Indeed, such structures would generate specular (mirror-like) iridescence with incident and reflected light rays exhibiting the same angle to the opposite sides of the surface normal. On the other hand, the observed backscattering (i.e., the light reflected by the egg sac surface comes back to the direction of the light source, rather than being reflected away) is compatible with the presence of either a surface diffraction grating (see Note 1, Fig. S2, ESI $\dagger$ ) or a photonic crystal in the bulk of the egg sac material.

\section{Fourier power analysis: from structure to optics}

We analysed the series of electronic images from FIB-SEM (Fig. 3) using a modified version of the Fourier tool from Prum and Torres. $^{26}$ We accounted for a dehydration shrinkage of about $18 \%$ and scaled accordingly the FIB-SEM images, generating an average length scale of about $230 \mathrm{~nm}$. As photography experiments indicate that the iridescent colour varies along the $X$-axis (see Fig. 4 and 5a), we used FIB-SEM images of $X Y$ planes to analyse the effects of illumination and observation angles on the backscattered Fourier power. We consider two

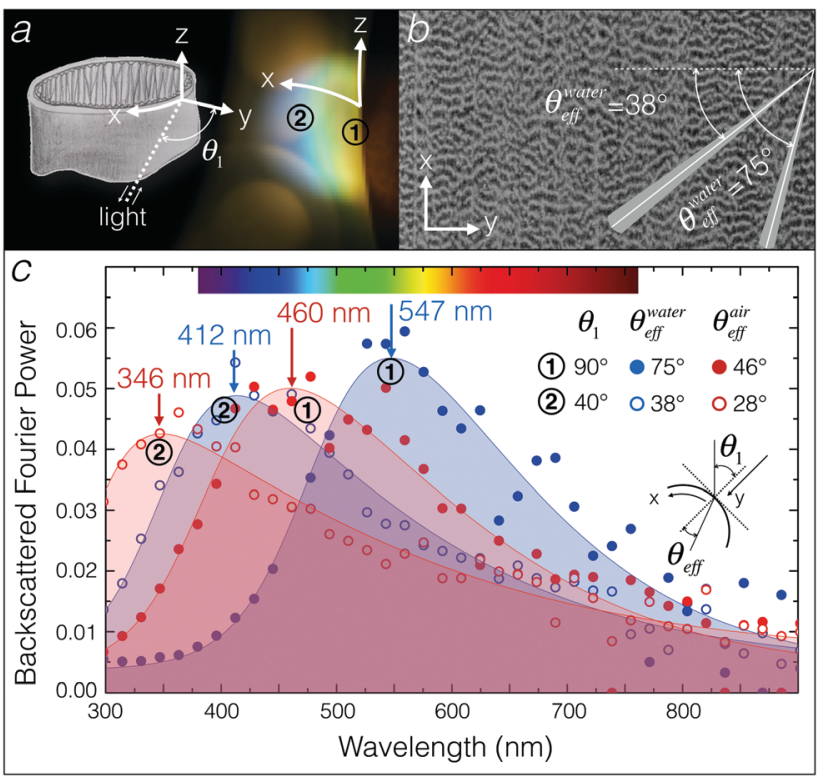

Fig. 5 (a) Geometry of the egg sac envelope (left panel); the incident light hits the envelope at an angle $\theta_{1}$ that varies from $90^{\circ}$ (grazing incidence on the border of the sac) to $0^{\circ}$ (perpendicular incidence in the middle of the sac); points (1) and (2) on the right panel (photograph of illuminated egg sac in water) correspond to angles of about $90^{\circ}$ and $40^{\circ}$. (b) Typical cropped image of a $X Y$ section; the angles indicated (shaded interval $=5^{\circ}$ tolerance) are those used in Fourier power spectrum analyses. (c) Backscattered Fourier power spectrum for angles of incidence corresponding to observation points (1) and (2) as the envelope is in water (blue fits) or in air (red fits); the inset shows a sketch of the angles $\theta_{1}$ (incident angle at which the light hits the envelope) and $\theta_{\text {eff }}$ (effective angle of incidence within the egg envelope material).

localisations on the inner curvature of the egg sac (Fig. 5a): (1) the edge of the envelope exhibiting a yellow-green colour and corresponding to an illumination/observation angle of about $90^{\circ}$, and (2) the zone exhibiting a blue colour and corresponding to an illumination/observation angle of about $40^{\circ}$. Note that we average the backscattered response across an angle of $5^{\circ}$ in the $X Y$ plane (Fig. $5 \mathrm{~b}$ ) in order to account for the effect of curvature variation in a finite observation spot size in the spectroscopy measurements. Finally, to integrate the disorder in the $Z$ direction, we average the backscattered Fourier power of $450 X Y$ FIB-SEM slices (Fig. 3a and Movie S1, ESI $\dagger$ ).

Because of the non-normal incidence at the interface between the external medium (water or air) and the egg envelope material ( $c f$. inset in Fig. $5 c$ ), the effective angle of incidence $\left(\theta_{\text {eff }}\right)$ on the periodic structure is defined by:

$$
\theta_{\text {eff }}=\sin ^{-1}\left(\frac{n_{1}}{n_{\text {eff }}} \sin \left(\theta_{1}\right)\right)
$$

where $n_{1}$ is the refractive index of the external medium $\left(n_{1}=1.33\right.$ or $n_{1}=1$, for water or air, respectively) and $n_{\mathrm{eff}}=$ $1.380 \pm 0.005$, i.e., the effective refractive index of the egg sac material as measured with a refractometer (ORA 1GG, Kern \& Sohn GmbH, Germany).

Fig. 5c shows the backscattered Fourier power spectrum for the two angles of incidence $\theta_{1}=90^{\circ}$ and $\theta_{1}=40^{\circ}$ in water (blue) 
and air (red); the corresponding effective angles of incidence $\left(\theta_{\text {eff }}\right)$ are indicated (Fig. 5c). Filled curves are asymmetric double sigmoidal peak fits of Fourier power distribution. Blue curves indicate an expected spectral blue shift as the incidence angle increases from point 1 to point 2, in good agreement with the observed colours (Fig. 5a). Changing the refractive index from $n_{1}=$ 1.33 (water) to $n_{1}=1$ (air), generates a blue shift of about $90 \mathrm{~nm}$ in wavelength for all angles of incidence (red data points and red curves in Fig. 5c), moving essentially most of the reflected structural colours outside of the visible range and, hence, explaining that the egg sac loses visible iridescence when removed out of the water. Note that this shift is unaffected by the presence of a layer of water at the surface of the egg sac as long as this layer is thin and of homogeneous thickness (see Fig. S3, ESI $\dagger$ ).

\section{Spectroscopy}

We recorded the backscattering spectra of the egg sac surface for various incident light angles $\theta_{1}$ in the $X Y$ plane (see Fig. S4a-c, $\mathrm{ESI} \dagger)$. As the spectrometer and the illumination source were connected with a bifurcated optical fiber, the incident light and recorded reflected light have the same orientation. When we vary the illumination angle along the $X$-axis (see Fig. S4a (ESI $\dagger$ ), left panel) of the egg sac submerged in water, the backscattered reflectance increases from about $10 \%$ to about $35 \%$ when the incident angle is increased from $30^{\circ}$ to $70^{\circ}$ (Fig. S4b, ESI $\dagger$ ). This observed relation, also captured by the backscattered Fourier power spectrum of FIB-SEM images (Fig. 5) for wavelengths $>450 \mathrm{~nm}$, cannot be easily anticipated because (i) backscattered reflectance is generated by an interference effect on a complex 3D structure and (ii) other phenomena, such as specular reflection on the egg sac interface as well as path length, absorption and incoherent scattering in the envelope material, can also vary for different incident angles, hence, can impact the observed reflectance. The relatively low reflectance intensity (about 20-35\%) might be due to some incident light being lost in other diffraction orders of the grating and by scattering within the structure. However, note that the part of the variation in the intensity recorded at different angles is likely due to the differences of distance between the probe and the sample surface. In particular, at grazing incidence $\left(\theta_{1}=80^{\circ}\right)$, the distance between the sample and the probe had to be substantially increased and only a portion of the sample was illuminated. In other words, the intensities of the curves shown in Fig. S4b (ESI $\dagger$ ) should not be given as much relevance as the shape of the corresponding spectra. At $80^{\circ}$, the spectrum maximum is at about $470 \mathrm{~nm}$, which corresponds to blue (almost green) light. At lower angles, the peak maximum shifts to yet shorter wavelengths and quickly exits the visible range.

When the egg sac is removed from water, the measured reflectance spectra have a low intensity and are nearly flat (see Fig. S4c, ESI $\dagger$ ). Additional measurements in the $Y Z$ plane (see Fig. S4a, right panel, ESI $\dagger$ ) indicate that the egg sac is not iridescent along its long $(Z)$ axis (see Fig. S4d, ESI $\dagger$ ).

\section{Biological function}

A discussion of the possible biological function(s) of the iridescence (including in the UV) of the salamander egg sac remains speculative; our goal is to direct potential future research to specifically test a set of alternative or perhaps complementary hypotheses. The presence of an iridescent egg sac seems restricted to Hynobiidae and Cryptobranchidae, the only salamanders (along with, possibly, the Sirenidae ${ }^{27}$ ) with external fertilisation; all other salamander species produce spermatophores and the eggs are fertilised internally (in the female's cloaca). ${ }^{28,29}$ Hence, one could speculate that iridescence is a functional character associated with external fertilisation. However, this argument is very weak because these two families are sister groups and split from the most basal node in the Caudata (salamanders) phylogeny, ${ }^{30}$ hence, the correlation observed between egg-sac iridescence and the species breeding habits might simply be due to a phylogenetic rather than a functional constraint.

In amphibians with external fertilisation, multiple paternity can improve fitness of the female by increasing the fertilisation rate, as observed in the European common frog Rana temporaria. ${ }^{31}$ In this respect, as salamanders' retina include rods sensitive to blue light ${ }^{32,33}$ and sperm can penetrate the egg sac jelly shortly after deposition, ${ }^{34}$ one could hypothesise that iridescent egg sacs attract additional males and increase the chances of multiple paternity and fertilisation rates. However, this hypothesis seems poorly compatible with the fact that scramble-competition of males over egg sacs only occurs ${ }^{35}$ in pond-breeding Hynobius dunni and H. nigrescens whereas stream-breeding Hynobius species (with particularly strong iridescence of their egg sacs) deposit a pair of egg sacs in dark places (under stones and in cavities) and do not present breeding aggregations. In addition, the variant of the blue-sensitive pigment that confers scotopic vision (i.e., colour vision in very dim light) to anurans (frogs and toads) ${ }^{33,36}$ as far as known is absent in salamanders. Much further analyses (behavioural assays, molecular genetics, molecular investigation of retinal pigments in species of stream-breeding Hynobius, etc.) are warranted to test the hypothesis of iridescence serving as a signal for increasing the probability of multiple paternity.

An alternative hypothesis is that the iridescence of the egg sac is a signal for predator deterrence, indicating unpalatability or toxicity (neither of the two were tested in these species) of the egg sac or eggs, or through exploitation of dietary conservatism and neophobia. ${ }^{37}$ Finally, a third hypothesis associated with a potential function of the egg sac iridescence is that it would act as an intra-specific display to avoid cannibalism; it has been reported that a female of $H$. kimura, in captivity, stayed near her eggs and drove approaching males away from the eggs at least 24 hours after oviposition. ${ }^{38}$

Importantly, given that the iridescence exhibited by the Hynobius salamander egg sacs is due to the material that makes up the envelope, it is possible that the observed structural colours are simply a non-functional by-product of selection acting on other characters. In other words, the envelope of the Hynobius egg sac (a character that is not observed in other amphibians ${ }^{39}$ ), might have been selected for its non-optical features (e.g., mechanical properties, barrier against pathogens and/or egg predators like planarians) brought about by structural characteristics that, incidentally, happen to generate iridescence. The predisposition 
for producing these structures may be phylogenetically conserved in the ancient salamander clade made up by hynobiids and cryptobranchids.

\section{Conclusions}

Here, we have presented the structural basis and physical principles explaining the observed blue to yellow iridescence of Hynobius kimurae egg sacs. Using electron microscopy, we first observed the presence of a potential diffraction grating (thin fibres of $190 \mathrm{~nm}$ length scale) on the inner surface of the envelope. We then performed TEM imaging of cross-sections of the envelope parallel or perpendicular to the elongated axis of the egg sac and confirmed the existence of quasi-periodical structural variation (fibres) of the material across the bulk of the egg sac envelope. Fourier-power analyses of FIB-SEM data then indicated the presence of a photonic structure with a quasi-periodic 1D modulation of 140 to $190 \mathrm{~nm}$ in the bulk of the envelope. Taking into account an approximate $18 \%$ shrinkage due to the preparation of samples for electron microscopy, ${ }^{40}$ this translates to a period of 170 to $230 \mathrm{~nm}$ in the fresh egg sac under atmospheric conditions. As discussed in the ESI $\dagger$ (see Note 1), this period also seems to vary depending on the localisation on the crescent-shape egg sac: smaller length scale on the outer curvature and larger length scale on the inner curvature. This trend is compatible with a mechanical origin of this variation because the periodicity is running parallel to the axis of the egg sac.

As the iridescence is present mostly on the sides of the envelope, i.e., at high incident illumination/observation angles, we expected the effect not to be specular. We tested this hypothesis by shining a light source and observing the variation of the optical response of the egg sac when changing the position of the sample or changing the orientation of the observer (camera). Both experiments indicate that constructive interference leading to structural colours is only observed in backscattering. These results rule out the possibility that the iridescence is due to a thin film or a multilayer. Additionally, our spectroscopic measurements confirm that the iridescence appears along the radius of the egg sac (while it is absent along the elongated axis) and is due to backscattering.

Next, we applied a Fourier-power analysis to our FIB-SEM data and showed that the predicted iridescence generated by these simulations matches the experimental photographic/spectroscopic data. Indeed, the Fourier-power modelling correctly predicts that (i) iridescence of the egg sac submerged in water spans the blue-to-yellow visible range of the light spectrum and (ii) the iridescence experiences a blue shift of about $90 \mathrm{~nm}$ (making it appear mostly in the UV) when the egg sac is removed out of the water. This spectral shift, resulting in the loss of visible iridescence, is due to a larger difference in refractive index between the air/egg-envelope versus water/egg-envelope interfaces. Note that the diffuse yellow hue of the egg sac in air is likely due to the absorption or scattering of blue wavelengths inside the material.

Finally, we have provided several hypotheses for the potential biological function of the observed iridescence (recognition of egg sacs by males for external fertilisation or display against predators or against intra-specific cannibalism), although we emphasize the possibility that iridescence is a non-functional by-product of the structural features of the egg sac envelope selected for their mechanical or anti-microbial properties. We note here the difficulties associated with the analysis of optical responses of soft materials because they tend to exhibit poor conservation properties (the material can alter with time) and their structural and photonic properties are easily perturbed during experimental procedures such as fixation and preparation for electron microscopy.

\section{Materials and methods}

\section{Material collected}

Fresh egg sacs of Hynobius kimurae were collected in early spring from a small headstream of the Nakatsu River (Kyoto Prefecture, Japan) and stored in distilled water for optical experiments or fixed (in $10 \%$ formalin or $2.5 \%$ glutaraldehyde) for electron microscopy.

\section{Photography}

We used a halogen light source (Ocean Optics, HL-2000) with an optical fibre (Ocean Optics, QP1000-2-VIS-BX) for illumination and a Nikon D800 camera for data acquisition. A fresh egg sac was placed in a black plastic bowl to avoid back scattering from the bottom of the recipient. The bowl was either left empty or filled with double distilled water for experiments in air or water, respectively. Distance between the camera and the egg sac was kept constant while the angle changed. The incident white light direction was fixed at $8^{\circ}$ from the vertical camera position.

\section{Scanning electron microscopy (SEM)}

A piece of a formalin-fixed egg sac was first dehydrated by critical point drying and then gold-coated before introduction into the vacuum chamber of a JEOL JSM-6510LV microscope using an electron beam energy of $10 \mathrm{kV}$ and a spot size of 30 .

\section{Transmission electron microscopy (TEM)}

Small rectangles (less than $1 \mathrm{~mm}^{2}$ ), cut from a piece of a glutaraldehyde-fixed egg sac, were post-fixed in $90 \mathrm{~min}$ at room temperature in a mixture of osmium tetroxide 1\% (EMS, Hatfield, PA, USA) with $1.5 \%$ of potassium ferrocyanide (Sigma, St Louis, MO, USA) in phosphate buffer. The samples were then washed three times with distilled water and dehydrated in solutions of acetone (Sigma, St Louis, MO, US) at graded concentrations (40 $\mathrm{min}$ in $30 \%$ acetone; $40 \mathrm{~min}$ in $70 \% ; 1 \mathrm{~h}$ in $100 \%$; and $2 \mathrm{~h}$ in $100 \%$ ). This was followed by the infiltration of Epon (Sigma, St Louis, MO, USA), with successive baths at graded concentrations ( $2 \mathrm{~h}$ in $1 / 3$ Epon/acetone; $2 \mathrm{~h}$ in $3 / 1$ Epon/acetone; then 1 to $4 \mathrm{~h}$ and $12 \mathrm{~h}$ in pure Epon), followed by $48 \mathrm{~h}$ of polymerisation in an oven at $60{ }^{\circ} \mathrm{C}$. Ultrathin transversal sections of $50 \mathrm{~nm}$ were cut with an Ultracut microtome (Leica Mikrosysteme GmbH, Vienna, Austria) and placed on $2 \times 1 \mathrm{~mm}$ copper slot grids (EMS, Hatfield, PA, USA) coated 
with a polystyrene film (Sigma, St Louis, MO, USA). Sections were post-stained for $10 \mathrm{~min}$ with $4 \%$ uranyl acetate (Sigma, St Louis, MO, USA) in water (and rinsed several times with water) and then incubated for $10 \mathrm{~min}$ with Reynolds lead citrate (and rinsed several times with water).

Data was acquired with a TVIPS TemCam-F416 digital camera (TVIPS GmbH, Gauting, Germany) mounted on a TEM Philips CM100 (Thermo Fisher Scientific, Waltham, MA USA) at an acceleration voltage of $80 \mathrm{kV}$. Large-scale montage stitching of high-resolution images was performed using the Blendmont command-line program (IMOD software ${ }^{41}$ ). 2D Fast Fourier Transform (FFT) analysis of the montage was performed in Fiji ImageJ (Version 2.0.0-rc-69/1.52p) ${ }^{42}$ using rectangular selection of the entire $X Y$ plane (from Fig. 2b) without the upper dense layer (from Fig. 2c).

\section{Focused ion beam scanning electron microscopy (FIB-SEM)}

FIB-SEM imaging was performed on the same resin block used for the TEM sections. The preparation regime of the block in the FIB-SEM microscope (FEI Company, Helios Nanolab 650 DualBeam) was performed as in Kizilyaprak et $a .^{43}$ The region of interest (ROI) was localised using the corresponding TEM images and a protective carbon layer of thickness $1 \mu \mathrm{m}$ was deposited on the block-surface of the ROI using a gas-injection system in the FIB-SEM instrument. To avoid re-deposition on the ROI during the milling process, trenches were milled on each side of the ROI with an ion beam current of $65 \mathrm{nA}$. The FIB-SEM tomography of the ROI was performed with the Slice and View software (FEI). The milling was performed with an accelerating voltage of $30 \mathrm{kV}$ at $790 \mathrm{pA}$ and $1 \mu \mathrm{s}$ dwell time corresponding to a slice thickness of $10 \mathrm{~nm}$ in the $Z$ direction. In other words, the sample was mounted in such a way that the ion beam cuts the transverse sections of the egg sac corresponding to the $X Y$ planes. Images of $3072 \times 2048$ pixels (corresponding to a pixel size of $3.9 \mathrm{~nm}$ ) were acquired by detecting backscattered electrons using an intra-column detector with an electron beam of $2 \mathrm{kV}$ at $800 \mathrm{pA}$ and $5 \mu$ s of imaging time with drift correction; each plane was imaged as the average of 4 acquisitions.

The movie representation of the FIB-SEM data was computed at $1280 \times 720$ dpi resolution with the AMIRA software (ThermoFisher Scientific, Waltham, MA, USA) and was annotated in the Filmora software (Wondershare, Shenzhen, China). Fourier transform analyses were performed in Fiji ImageJ (Version 2.0.0-rc-69/1.52p). ${ }^{42}$

\section{Refractometry}

The effective refractive index of the egg sac material was measured using an analogue refractometer (KERN \& SOHN GmbH, Ora 1 GG, Germany) with a scale accuracy of 0.01 . The surface of the prism was wetted with a drop of 1-Bromnaphtalene (refractive index 1.657) prior to the measurement for optical coupling. A small piece of the egg sac $(1 \times 1 \mathrm{~cm})$ was wiped to remove the residual water and placed flat onto the prism. After closing the prism cover, the value of the egg sac's refractive index was read directly from the scale.

\section{Spectroscopy}

Spectroscopic measurements were performed on a $2 \mathrm{~cm}^{2}$ piece of egg sac that was partially cleaned from mucus and maintained in double-distilled water for a year. The piece was placed into a Petri dish filled with water (or air). To avoid backscattering from the bottom of the Petri dish, the surface of the dish was covered with a black sheet of plastic. The light from a halogen lamp (HL-2000, Ocean Optics, Largo, FL, USA) was focused to the central channel of a bifurcated probe (QR400-7-VIS-NIR, Ocean Optics) allowing immersed measurements. The reflected light was collected by surrounding optical fibres and focused into a spectrometer (HR 2000+, Ocean Optics).

\section{Conflicts of interest}

There are no conflicts to declare.

\section{Acknowledgements}

We thank Caroline Kizilyaprak, Jean Daraspe, and Bruno Humbel from the Electron microscopy facility of the University of Lausanne for the assistance with TEM and FIB-SEM. We thank Peter Vukusic for commenting on data and analyses. This work was supported by grants to MCM from the Swiss National Science Foundation (FNSNF, grants CRSII3_154406, CR32I3_162743), the Georges \& Antoine CLARAZ foundation, the SystemsX.ch initiative (project EpiPhysX) and the International Human Frontier Science Program Organisation (HFSP RGP0019/ 2017). SRM salary was supported by the Belgian National Fund for Scientific Research (FRS-FNRS; 91400/1.B.309.18F). The funding bodies played no role in the design of the study, collection, analysis, and interpretation of data and in writing the manuscript.

\section{References}

1 M. F. Land, Prog. Biophys. Mol. Biol., 1972, 24, 75-106.

2 P. Vukusic and J. R. Sambles, Nature, 2003, 424, 852-855.

3 S. Kinoshita and S. Yoshioka, ChemPhysChem, 2005, 6, 1442-1459.

4 S. Kinoshita, S. Yoshioka and J. Miyazaki, Rep. Prog. Phys., 2008, 71, 076401.

5 J. Huang, X. Wang and Z. L. Wang, Nano Lett., 2006, 6, 2325-2331.

6 S. A. Morin, R. F. Shepherd, S. W. Kwok, A. A. Stokes, A. Nemiroski and G. M. Whitesides, Science, 2012, 337, 828-832.

7 Y. J. Zhao, Z. Y. Xie, H. C. Gu, C. Zhu and Z. Z. Gu, Chem. Soc. Rev., 2012, 41, 3297-3317.

8 H. Wang and K. Q. Zhang, Sensors, 2013, 13, 4192-4213.

9 G. England, M. Kolle, P. Kim, M. Khan, P. Muñoz, E. Mazur and J. Aizenberg, Proc. Natl. Acad. Sci. U. S. A., 2014, 111, 15630-15634.

10 H. W. Yin, L. Shi, J. Sha, Y. Z. Li, Y. H. Qin, B. Q. Dong, S. Meyer, X. H. Liu, L. Zhao and J. Zi, Phys. Rev. E: Stat., Nonlinear, Soft Matter Phys., 2006, 74, 051916.

11 M. Kurachi, Y. Takaku, Y. Komiya and T. Hariyama, Naturwissenschaften, 2002, 89, 295-298. 
12 D. G. Stavenga, B. D. Wilts, H. L. Leertouwer and T. Hariyama, Philos. Trans. R. Soc. London, Ser. B, 2011, 366, 709-723.

13 D. Gur, B. Leshem, M. Pierantoni, V. Farstey, D. Oron, S. Weiner and L. Addadi, J. Am. Chem. Soc., 2015, 137, 8408-8411.

14 A. L. Ingram, V. Lousse, A. R. Parker and J. P. Vigneron, J. R. Soc., Interface, 2008, 5, 1387-1390.

15 J. P. Vigneron, P. Simonis, A. Aiello, A. Bay, D. M. Windsor, J.-F. Colomer and M. Rassart, Phys. Rev. E: Stat., Nonlinear, Soft Matter Phys., 2010, 82, 021903.

16 D. S. Dhillon, J. Teyssier, M. Single, I. Gaponenko, M. C. Milinkovitch and M. Zwicker, Computer Graphics Forum, 2014, 33, 177-188.

17 S. Vignolini, P. J. Rudall, A. V. Rowland, A. Reed, E. Moyroud, R. B. Faden, J. J. Baumberg, B. J. Glover and U. Steiner, Proc. Natl. Acad. Sci. U. S. A., 2012, 109, 15712-15715.

18 S. Vignolini, T. Gregory, M. Kolle, A. Lethbridge, E. Moyroud, U. Steiner, B. J. Glover, P. Vukusic and P. J. Rudall, J. R. Soc., Interface, 2016, 13, 20160645.

19 K. Michielsen and D. G. Stavenga, J. R. Soc., Interface, 2008, 5, 85-94.

20 J. Teyssier, S. V. Saenko, D. van der Marel and M. C. Milinkovitch, Nat. Commun., 2015, 6, 6368.

21 I. Sato, A Monograph of the Tailed Batrachians of Japan, Nippon Shuppan-sha, Osaka, 1943.

22 M. Rassart, et al., New J. Phys., 2008, 10, 033014.

23 S. R. Mouchet, M. Lobet, B. Kolaric, A. M. Kaczmarek, R. Van Deun, P. Vukusic, O. Deparis and E. V. Hooijdonk, Proc. R. Soc. B, 2016, 283, 20162334.

24 C. J. Chandler, B. D. Wilts, S. Vignolini, J. Brodie, U. Steiner, P. J. Rudall, B. J. Glover, T. Gregory and R. H. Walker, Sci. Rep., 2015, 5, 11645.

25 M. Matsui and S. Seki, The Handbook of Tadpoles, Bun-Ichi Co, Tokyo, 2008.

26 R. O. Prum and R. H. Torres, Integr. Comp. Biol., 2003, 43, 591-602.

27 S. Reinhard, S. Voitel and A. Kupfer, Zool. Anz., 2013, 253, $1-5$.
28 R. C. R. Bruce, Herpetologica, 2003, 59, 301-310.

29 S. Kuehnel, S. Reinhard and A. Kupfer, Bonn Zoological Bulletin, 2010, 57, 119-126.

30 I. Irisarri, D. Baurain, H. Brinkmann, F. Delsuc, J. Y. Sire, A. Kupfer, J. Petersen, M. Jarek, A. Meyer, M. Vences and H. Philippe, Nat. Ecol. Evol., 2017, 1, 1370-1378.

31 D. R. Vieites, S. Nieto-Roman, M. Barluenga, A. Palanca, M. Vences and A. Meyer, Nature, 2004, 431, 305-308.

32 Y. Takahashi, O. Hisatomi, S. Sakakibara, F. Tokunaga and Y. Tsukahara, FEBS Lett., 2001, 501, 151-155.

33 K. Kojima, Y. Matsutani, T. Yamashita, M. Yanagawa, Y. Imamoto, Y. Yamano, A. Wada, O. Hisatomi, K. Nishikawa, K. Sakurai and Y. Shichida, Proc. Natl. Acad. Sci. U. S. A., 2017, 114, 5437-5442.

34 M. Ohta, H. Kubo, Y. Nakauchi and A. Watanabe, Zool. Sci., 2010, 27, 875-879.

35 M. Hasumi, J. Herpetol., 1994, 28, 264-267.

36 C. A. M. Yovanovich, S. M. Koskela, N. Nevala, S. L. Kondrashev, A. Kelber and K. Donner, Philos. Trans. R. Soc., B, 2017, 372, 20160066.

37 N. M. Marples, D. J. Kelly and R. J. Thomas, Evolution, 2005, 59, 933-940.

38 E. Fukuta, R. Iwaki, S. Murase, N. Kato and H. Miyake, Bulletion of the Herpetological Society of Japan, 2019, 2019, 28-31.

39 R. Altig and R. W. McDiarmid, Herpetological Monographs, 2007, 21, 1-32.

40 F. A. Shah, B. R. Johansson, P. Thomsen and A. Palmquist, J. Biomed. Mater. Res., Part A, 2015, 103, 1565-1576.

41 J. R. Kremer, D. N. Mastronarde and J. R. McIntosh, J. Struct. Biol., 1996, 116, 71-76.

42 J. Schindelin, I. Arganda-Carreras, E. Frise, V. Kaynig, M. Longair, T. Pietzsch, S. Preibisch, C. Rueden, S. Saalfeld, B. Schmid, J.-Y. Tinevez, D. J. White, V. Hartenstein, K. Eliceiri, P. Tomancak and A. Cardona, Nat. Methods, 2012, 9, 676-682.

43 C. Kizilyaprak, G. Longo, J. Daraspe and B. M. Humbel, J. Struct. Biol., 2015, 189, 135-146. 